\title{
Assessing lesion malignancy by scanning small-angle $X$-ray scattering of breast tissue with microcalcifications
}

\author{
C. Arboleda, ${ }^{1,2, a)}$ V. Lutz-Bueno, ${ }^{1, b)}$ Z. Wang, ${ }^{1,2}$ P. Villanueva-Perez, ${ }^{1}$ M. Guizar-Sicairos, ${ }^{1}$ M. Liebi, ${ }^{3,4}$ Z. \\ Varga, ${ }^{5}$ and M. Stampanoni ${ }^{6,2}$ \\ 1) Swiss Light Source, Paul Scherrer Institute, 5232 Villigen, Switzerland \\ ${ }^{2)}$ ETH Zurich, 8092 Zurich, Switzerland \\ 3) MAX IV Laboratory, 22592 Lund, Sweden \\ 4) Chalmers University of Technology, 41258 Goeteborg, Sweden \\ ${ }^{5)}$ Institute of Pathology and Molecular Pathology, University Hospital Zurich, 8091 Zurich, \\ Switzerland \\ 6) PSI, 5232 Villigen, Switzerland
}

(Dated: 15 August 2019)

\begin{abstract}
Scanning small-angle X-ray scattering (SAXS) measurements were performed on 36 formalin-fixed breast tissue biopsies, obtained from two patients. All samples contained microcalcifications of type II, i.e. formed by hydroxyapatite. We demonstrate the feasibility of classifying breast lesions by scanning SAXS of tissues containing microcalcifications with a resolution of $35 \mu \mathrm{m} \times 30 \mu \mathrm{m}$. We report a characteristic Bragg peak found around $\mathrm{q}=1.725 \mathrm{~nm}^{-1}$ that occurs primarily for malignant lesions. Such a clear SAXS fingerprint is potentially linked to structural changes of the breast tissue and correspond to dimensions of about $3.7 \mathrm{~nm}$. Such a material property could be used as an early indicator of malignancy development, as it is readily assessed by SAXS. If this fingerprint is combined with other known SAXS features, which also indicate the level of malignancy, such as lipid spacing and collagen periodicity, it could complement traditional pathologybased analyses. To confirm the SAXS-based classification, a histopathological workup and a gold standard histopathological diagnosis were conducted to determine the malignancy level of the lesions. Our aim is to report this SAXS fingerprint, which is clearly related to malignant breast lesions. However, any further conclusion based on our dataset is limited by the low number of patients and samples. Running a broad study to increase the number of samples and patients is of great importance and relevance for the breast-imaging community.
\end{abstract}

Keywords: Small-angle X-ray scattering, breast cancer, breast microcalcifications, breast lesion diagnosis

\section{INTRODUCTION}

Breast cancer is the second most commonly diagnosed ${ }^{52}$ cancer among women after skin cancer $^{1}$. Even though 53 several in situ and in vivo imaging methods are com- 54 bined to increase the confidence of cancer malignancy 55 level assessment, the accurate diagnosis of cancer remains 56 a challenge and biopsy extraction is often required to 57 confirm whether a breast tissue lesion is benign or ma- 58 lignant. Cancer diagnosis and treatment require a multi- 59 disciplinary team composed of surgeons, pathologists and 60 technicians, to have full access to the clinical history of 61 the patient as well as the biological and structural char- 62 acteristics of the biopsy sample.

One important predictor of breast cancer is the pres- 64 ence of microcalcifications, which are usually benign de- 65 posits of calcium. Orsi et al. ${ }^{2}$ suggested that the mor- 66 phology and distribution of microcalcifications may indi- 67 cate the malignancy level of breast tissue lesions. Grating 68 interferometry (GI) has been tested on its ability to pro- 69 vide a non-invasive malignancy level assessment based on 70 the microcalcifications type and structure ${ }^{3-5}$. The results 71

a) Electronic mail: carolina.arboleda@psi.ch

b) Electronic mail: Viviane.Lutz-Bueno@psi.ch
50 of those investigations suggest that measuring scattering interactions can potentially discriminate morphological characteristics that are associated to cancer ${ }^{4-6}$.

Insights into the structural organization of biological tissues provide ways to understand how their processes, diseases and functionality develop. Small-angle X-ray scattering (SAXS) is able to provide structural information of noncrystalline materials in the range of tens of micrometers down to a few nanometers ${ }^{7,8}$. If a scanningbased SAXS setup is combined with a focused X-ray beam, two-dimensional maps of the structural changes can be obtained for heterogeneous samples ${ }^{9-14}$. For instance, Siu et al. ${ }^{15}$ evaluated the diagnostic potential of SAXS for brain tumors and concluded that this technique can be an effective classifier of malignancy. Giannini et al. ${ }^{16}$ evaluated the SAXS and wide-angle X-ray scattering (WAXS) patterns of bone biopsies collected from osteoarthritis-affected patients. After performing principal component and canonical correlation analyses on the SAXS and WAXS data, the nanostructural properties of collagen over extended areas of bone were characterized and quantified. They concluded based on structural changes that there is correlation between age and cross-linking-induced rigidity of collagen fibers.

Among the numerous diseases that provoke structural changes in tissues, the understanding of the formation and the classification of breast cancer with high accuracy

This document is the accepted manuscript version of the following article: Arboleda, C., Lutz-Bueno, V., Wang, Z., Villanueva-Perez, P., Guizar-Sicairos, M., Liebi, M., ... stampanoni, M. (2019). Assessing lesion malignancy by scanning small-angle x-ray scattering of breast tissue with microcalcifications. Physics in Medicine and Biology, 64(15), 155010 (9 pp.). https://doi .org/10.1088/1361-6560/ab2c36 
is of fundamental importance. Fernandez et al. ${ }^{10}$ em-131 ployed SAXS to investigate 28 human breast tumor specimens. Differences between the scattering signals from invasive malignant lesions and healthy regions were ob-132 served. Ryan and Farquharson ${ }^{17}$ measured Compton and coherent scattering interactions, using an X-ray tube and ${ }_{133}$ a high-purity Germanium (HPGe) detector, to classify ${ }_{134}{ }^{133}$ malignant and benign breast tissue lesions. The scatter- ${ }_{135}$ ing spectra were analyzed with a peak-fitting routine $\operatorname{and}_{136}$ a subsequent multivariate analysis. A sensitivity of $54 \%_{137}$ and a specificity of $100 \%$ were obtained for the dataset. ${ }_{138}$ Despite valuable outcomes in breast cancer diagnosis by ${ }_{139}$ SAXS, to our knowledge none of them focused specifically ${ }_{140}$ on tissue containing microcalcifications, whose presence ${ }_{141}$ is known to constitute one of the most important diag- ${ }_{142}$ nostic markers of breast cancer ${ }^{18-21}$

Here we focus on 36 formalin-fixed human breast tis- ${ }^{143}$ sue samples from two different patients, all containing ${ }_{145}$ microcalcifications. Scanning SAXS measurements were ${ }_{146}$ performed using a focused X-ray beam with a resolution ${ }_{147}$ of $35 \mu \mathrm{m} \times 30 \mu \mathrm{m}$. The SAXS patterns distributed over ${ }_{148}$ millimetric areas of tissue containing embedded microcal- ${ }_{149}$ cifications served as a base for malignancy level assess- ${ }_{150}$ ment. Thanks to the spatial resolution and coverage pro- ${ }_{151}$ vided by scanning SAXS, different sample regions can be ${ }_{152}$ resolved. The possibility of distinguishing different types ${ }_{153}$ and structures of tissues in regions of a single sample en- ${ }_{154}$ ables a detailed analysis and makes easier to establish $\mathrm{a}_{155}$ link between malignancy and structural changes of the healthy tissue. Such reliable method could be a valuable candidate to increase the precision of cancer diag- ${ }_{156}$ nosis and classification. The outcomes of our SAXS data analysis indicate that a diagnosis based on the structural changes of a tissue that contains a microcalcification is ${ }^{157}$ promising in the examined range of scattering vectors ${ }^{158}$ $\mathrm{q}=0.5-4 \mathrm{~nm}^{-1}$, especially around $\mathrm{q}=1.725 \mathrm{~nm}^{-159}$ where a fingerprint was observed. However, it is neces- ${ }^{160}$ sary to increase the number of samples and patients to ${ }^{161}$ verify the relevance of the aforementioned findings.

\section{MATERIALS AND METHODS}

\section{A. Sample preparation}

Formalin-fixed human breast tissue samples from two 170 patients were attained from the Institute of Pathol-171 ogy and Molecular Pathology at the University Hospital 172 Zurich. Ethical consent was obtained from both patients.173 Radiographic measurements were performed to verify if $f_{174}$ microcalcifications were present. Thin $1-2 \mathrm{~mm}$ slices 175 were cut in the regions of interest (ROI) where micro-176 calcifications were observed. These slices were further ${ }_{177}$ imaged on a setup with a field of view of $10 \mathrm{~cm} \times 5 \mathrm{~cm}_{178}$ to select smaller ROIs. In total, 36 slices containing mi-179 crocalcifications were obtained. For scanning SAXS mea-180 surements, these slices were fixed on a sample holder us-181 ing Kapton foil and tape and keeping them embedded in ${ }_{182}$ formalin.

\section{B. Scanning SAXS measurements}

Scanning SAXS $^{9}$ was performed at the cSAXS beamline of the Paul Scherrer Institute. A monochromatic beam of $11.2 \mathrm{keV}(\lambda=1.107 \AA)$ was focused to about $35 \mu \mathrm{m} \times 30 \mu \mathrm{m}$. For every point in the raster scan, a SAXS pattern was recorded; at the same time, the sample transmission was measured by a diode at the beam stop. Scattering patterns were acquired using a PILATUS $2 \mathrm{M}^{22}$ detector placed $2.16 \mathrm{~m}$ away from the sample. An evacuated flight tube was placed between the sample and detector to reduce absorption and parasitic scattering from air. For fast acquisition, SAXS patterns were recorded in a continuous line scan mode with the sample moving at constant speed along $y$, while the detector continuously records data (Fig. 1a). The detector was operated with $35 \mathrm{~ms}$ exposure time and $5 \mathrm{~ms}$ readout time, i.e. with a frame rate of $25 \mathrm{~Hz}$. The total scan time was 19.6 hours for all 36 samples $(1.5 \mathrm{~cm} \times 1.5 \mathrm{~cm}$ in area each), with an X-ray flux of $1.81 \times 10^{14}$ photons $/ \mathrm{s} / \mathrm{mm}^{2}$. After these measurements, samples underwent a pathological workup with eosin/hematoxylin to assess their malignancy level ${ }^{23}$. Additionally, the microcalcification chemical type was evaluated based on its birefringence properties $^{24-26}$.

\section{Scanning SAXS data analysis}

The analysis of the data set composed of 36 samples was based on the presence of characteristic peaks, here called fingerprints, in the integrated scattering curves for the accessed experimental q-range of $0.5-4 \mathrm{~nm}^{-1}$, as shown in Fig. 1b. Our goal is to classify the samples in the data set in malignant and benign lesions. We name the samples as $\mathrm{b} i \mathrm{p} j$ and $\mathrm{m} i \mathrm{p} j$, where b stands for benign and $\mathrm{m}$ for malignant lesions, while $i$ is the sample number and $j$ is the patient number. As an example, we select sample m9p1. Figure 1b shows four selected scattering curves covering the whole measured q-range, while Fig. 1c zooms in the q-range $=1.67-1.75 \mathrm{~nm}^{-1}$ where fingerprints were observed. The location of the microcalcifications is clear from the transmission map in Fig. 1d, as the X-ray beam is less transmitted when hitting a microcalcification. Points S1-S4 indicate the positions where the scattering patterns in Fig. $1 \mathrm{~b}$ and $\mathrm{c}$ were measured. Points S1 and S4 are similar as they lay in tissue. As expected from breast tissue ${ }^{27}$, a prominent lipid Bragg peak is measured at $\mathrm{q}=1.5 \mathrm{~nm}^{-1}$ in all samples (see Figs. 1c) ${ }^{28}$. Point S2 hits the microcalcification and higher scattering intensities are measured over the whole q-range. The SAXS fingerprint discussed in this work is observed in S2 and S3, indicating that it occurs not only in regions near, but also in regions far away from the microcalcification. In this example, we compare the az- 
imuthally integrated SAXS patterns in regions near the $e_{238}$ calcification, from which the scattering curve S3 origi-239 nates displaying a clear peak around $1.725 \mathrm{~nm}^{-1}$. Note 240 that S2 also contains a peak around $1.725 \mathrm{~nm}^{-1}$, but it 241 has a higher baseline due to the high scattering origi-242 nated by the microcalcification. We restricted our peak ${ }_{243}$ finding routine to the q-range $=1.67-1.75 \mathrm{~nm}^{-1}$, as 244 shown in Fig. 1c, to avoid lipid and collagen peaks. $\operatorname{In}_{245}$ this range, we search for the position in $q$ and amplitude 246 A of peaks in the selected q-range by fitting a one-term 247 Gaussian function after baseline subtraction. We search ${ }_{248}$ for such peaks in all collected scattering patterns, there-249 with obtaining a map of their amplitude in each pixel 250 and sample, as shown in Fig. 1e.

The measured breast tissue lesions were classified 252 based on the histopathological diagnosis. From the 36253 measured samples, 21 were diagnosed as benign and 15 as254 malignant lesions. Regarding the chemical composition255 of the microcalcification, all were classified as hydroxya-256 patite or type $\mathrm{II}^{24}$. The histogram in Fig. If shows the257 distribution of peaks in the selected q-range for the 21258 benign samples, while Fig. $1 \mathrm{~g}$ shows the same for the 15259 malignant lesions. Each pixel is assigned a value of $1_{260}$ if there is a peak, and 0 if there is none. These pixel 261 labels are summed up for all benign or malignant sam-262 ples for each q-range shown in the histograms. It is clear263 that the large majority of pixels with such signature peak264 occurs for malignant samples in the searched q-range.265 Even though q has a distribution of peak positions in 266 Fig. 1g, the maximum number of peaks is found around 267 $1.725 \mathrm{~nm}^{-1}$, and this is the value we refer to as the fin-268 gerprint.

\section{RESULTS AND DISCUSSION}

For further discussion, the samples were separated $^{274}$ into two groups, benign and malignant, according to the $\mathrm{e}^{275}$ histopathological diagnosis. They were not further cate- ${ }^{276}$ gorized according to the cancer type or malignancy level, ${ }^{277}$ due to the reduced number of samples and patients. The ${ }^{278}$ main goal of this study is to test the ability of scan ${ }^{279}$ ning SAXS to discriminate between benign and malig- ${ }^{280}$ nant lesions. The transmission maps, as well as the ${ }^{281}$ peak amplitude maps, as previously discussed in Fig. $1{ }^{282}$ are shown for benign and malignant lesions in Figs. $2^{283}$ and 3 , respectively. We confirmed that all samples con- ${ }^{284}$ tain microcalcifications, based on the transmission maps ${ }^{285}$ shown in Figs. 2a and 3a. Clear microcalcifications are observed in the benign samples shown in Fig. 2a, although the amplitude of peaks in the q-range $=1.67$ $1.75 \mathrm{~nm}^{-1}$ do not form clear regions, neither near nor far from the microcalcifications, Fig. 2b, except for sample b21p2. An obvious difference is seen in Fig. 3. By comparing Figs. 3a and 3b, a feature emerges as a Bragg peak around $\mathrm{q}=1.725 \mathrm{~nm}^{-1}$ with large amplitude. Such features are spatially distributed near and far from the 286 microcalcifications. This fingerprint is associated to most 287 of the malignant samples, except for $\mathrm{m} 1 \mathrm{p} 1, \mathrm{~m} 2 \mathrm{p} 2, \mathrm{~m} 3 \mathrm{p} 2$, $\mathrm{m} 4 \mathrm{p} 2$ and m5p2. Such a clear spatially-resolved feature, which was revealed without any staining or human interpretation, since it is originated directly from structural changes of the tissue, can be potentially used as a fingerprint for cancer diagnosis. The amplitude maps of the Bragg peaks around $\mathrm{q}=1.725 \mathrm{~nm}^{-1}$ indicate that structural changes occur to the tissue near and far from the microcalcifications. However, whether the microcalcifications lead to the tissue structural changes or vice versa, remains unclear. Additionally, the fingerprint was absent in 5 out of the 15 malignant samples, which might be related to the corresponding malignancy level (BI-RADS) ${ }^{2}$. Although this fingerprint appeared in samples from both patients, which suggests that it may be patient independent, it is important to recall that the majority of benign specimens belonged to patient 2 , whereas most of the malignant samples originated from patient 1 . This constitutes a major limitation of this study and more patients are definitely needed to be able to reach a significant conclusion concerning the relevance of this fingerprint.

It can be observed in Fig. 3b that, whereas the top row samples m1-m5 do not display any clear peak amplitude in that q-range, all the other samples exhibit increased peak amplitudes located close to the microcalcifications. Samples m6p1 and m7p1 present peak amplitudes mostly located around the microcalcifications and nowhere else in the tissue, whereas the remaining eight samples display peaks even where no microcalcifications are found. One hypothesis is that the Bragg peak at $\mathrm{q}=1.725 \mathrm{~nm}^{-1}$ is a consequence of tissue structural remodeling induced by cancer and that the microcalcifications are somewhat responsible for this process. The reason why some microcalcifications do not generate such structural change in the tissue, as it is the case for samples m1-m5 as well as for the benign ones, is a potential study that could correlate this fingerprint with microcalcification nanostructure and morphology.

Two receiver-operating characteristic (ROC) curves $^{29}$ were calculated, shown in Fig. 4, to find the best threshold to classify benign and malignant lesions using either the presence of peaks or the sum of peak amplitudes per sample (Fig. 2d and 2e) in the $\mathrm{q}=1.67-1.75 \mathrm{~nm}^{-1}$ range as metric. In the former case, the pixel value was set to 1 where a peak was found and 0 otherwise. Afterwards, the sensitivity, also known as true positive rate (TPR), and specificity, also known as true negative rate (TNR), were calculated as follows:

$$
\begin{aligned}
& T P R=\frac{T P}{P}, \\
& T N R=\frac{T N}{N},
\end{aligned}
$$

where TP, P, TN and N, are the true positives, positives, true negatives and negatives, respectively. The Youden 

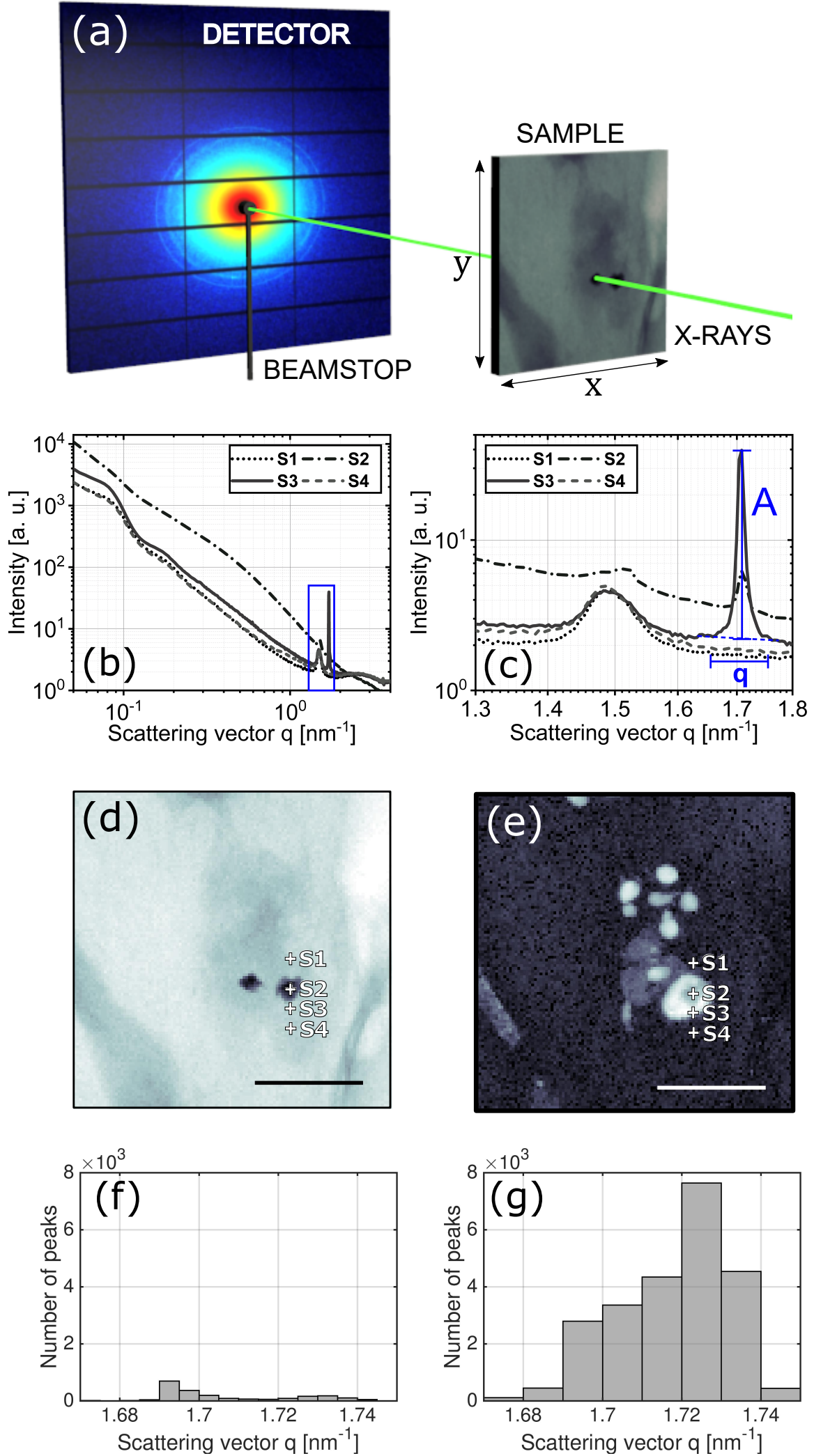

FIG. 1. (a) Experimental setup: The sample is scanned across an X-ray beam along $x$ and $y$. Scattering patterns are measured by a 2D detector at each scan point. The beamstop is equipped with a diode to simultaneously measure the sample's transmission. (b) Azimuthal integration of points S1-S4 covering the experimental q-range. (c) Zoom in the q-range = 1.3 $1.8 \mathrm{~nm}^{-1}$, where the amplitudes $\mathrm{A}$ of the peaks were extracted in the indicated q-range $=1.67-1.75 \mathrm{~nm}^{-1}$, after baseline subtraction. (d) Transmission map of sample m9p1. (e) Map of the amplitude of the peaks in the q-range $=1.67-1.75 \mathrm{~nm}^{-1}$ of sample m9p1. Points S1-S4 indicate the location of the scattering patterns shown in (b). (f) Histogram of all occurrences of peaks in the q-range $=1.67-1.75 \mathrm{~nm}^{-1}$ for the 21 benign lesion samples. (g) Histogram of all occurrences of peaks in the q-range $=1.67-1.75 \mathrm{~nm}^{-1}$ for the 15 malignant lesion samples. The scale bar corresponds to $1 \mathrm{~mm}$. 
index (sensitivity+specificity-1) ${ }^{29}$ was used to define the 340 optimal cutoff values in both cases and the outcomes are 341 summarized in table I.

TABLE I. Sensitivity and specificity at the optimal cutoff ${ }^{344}$ value (Youden index)

\begin{tabular}{ccc}
\hline Method & Sensitivity & Specificity \\
\hline Peak presence & $60 \%$ & $100 \%$ \\
Sum of peak amplitudes & $67 \%$ & $100 \%$ \\
\hline
\end{tabular}

The specificity of $100 \%$ generated by the presence of a peak at $\mathrm{q}=1.725 \mathrm{~nm}^{-1}$ indicates a high potential for ${ }^{349}$ breast cancer diagnosis for samples containing microcalcifications. The obtained specificity value bespeaks that 350 there is no risk of false positives, while the sensitivity 351 one indicates that up to $40 \%$ of the cancerous cases will 352 be missed if only the peak-presence detection criterion is ${ }^{353}$ employed. If the sum of peak amplitudes is considered ${ }^{354}$ instead, a $7 \%$ increase in sensitivity can be obtained. 355

The structural changes related to this SAXS finger-356 print could be related to the formation of fibrous tissue. ${ }^{357}$ It is known that healthy breast tissue is formed either 358 by evenly dispersed or well-ordered cells. In either case, 359 there are no extraneous proliferation or foreign materials 360 present. A cancerous tumour is formed when excessive 361 accumulation of abnormal cells occur. In vivo Magnetic ${ }^{362}$ Resonance Spectroscopy (MRS) studies ${ }^{31-33}$ showed that ${ }^{363}$ the water-to-fat ratio was higher in invasive ductal car-364 cinomas compared to benign lesions or normal breast 365 parenchyma $^{31,33,34}$. An ex-vivo Nuclear Magnetic Reso-366 nance (NMR) investigation further confirmed this finding 367 by demonstrating that malignant carcinosarcoma tissue ${ }^{368}$ has higher amounts of water content, which is directly ${ }^{369}$ related to fibrous tissue, compared to normal healthy 370 tissue $^{30}$. Since cancerous regions have the tendency to ${ }^{371}$ contain more fibrous tissue and water, the peak around ${ }^{372}$ $\mathrm{q}=1.725 \mathrm{~nm}^{-1}$ could correspond to such a structural ${ }^{373}$ change $^{17}$, and it might explain why this peak is only pre-374 dominant in malignant lesions.

The diagnosis based on the fingerprint at 376 $\mathrm{q}=1.725 \mathrm{~nm}^{-1}$ is only known for samples containing 377 microcalcifications, and it could be complementary of 378 other SAXS fingerprints previously reported. Most379 SAXS studies of human tissues relate abnormalities on 380 collagen structure to the level of malignancy of tumors.381 For example, the degradation of collagen is associated 382 with invasive carcinoma in breast tissue. This exhibits383 itself as disperse collagen bundles that break the order of 384 normal tissue. Collagen fibril degradation is associated 385 to the presence of an acidic collagen component that386 causes collagen chain modifications ${ }^{35-37}$. The collagen 387 degradation is seen in SAXS signals as the axial peaks388 of collagen become less intense and broader, due to theзs9 decrease of ordering with malignancy ${ }^{38}$. Another knownз90 SAXS fingerprint is the longitudinal arrangement of 391 collagen fibrils, which gives rise to axial d-spacing peaks392 that can be used to classify breast tissue types ${ }^{39-43} .393$ Small displacements of the position of the lipid Bragg $_{394}$ peak along q was indicated as a possible SAXS fingerprint for breast cancer diagnosis by Castro et al. ${ }^{28}$. Combining collagen, lipids and fibrous tissue SAXS fingerprints could expand the reliable tools available for cancer diagnosis, as well as level of malignancy assessment (BI-RADS) ${ }^{2}$. If scanning SAXS could be employed not only for classification, but also for BI-RADS categorization, it could become an excellent complement to histo-pathological workups.

\section{CONCLUSION}

We analyzed a set of 36 formalin-fixed human breast tissue samples from two different patients by scanning SAXS. The search and classification of fingerprints indicate that it might be possible to classify benign and malignant lesions based on the scattering signals from tissues containing microcalcifications. The presence of a Bragg peak around $\mathrm{q}=1.725 \mathrm{~nm}^{-1}$ generated a specificity of $100 \%$ and a sensitivity of up to $67 \%$ when the sum of peak amplitudes is considered per sample, to classify between benign and malignant lesions. Notwithstanding, it is important to recall that a limited number of samples from only two different patients were measured and that the majority of the malignant samples originated from one of the patients, which implies that more samples and patients are definitely required to reach more solid conclusions, including malignant lesions without microcalcifications. It is important to emphasize the added benefit of the spatial-resolution from scanning SAXS for the differentiation of the structural changes of tissue upon the growth of a tumor around a microcalcification. The fact that the scattering is not integrated over the whole sample volume allows discrimination between different sample regions. After this report, deeper investigations to link such a fingerprint to structural changes in breast tissue, as well as the BI-RADS level of cancer, would be essential to understand the development of cancer and its dependency on microcalcifications. Nevertheless, a potential SAXS fingerprint has been identified in malignant breast tissue lesions, which might offer potential in diagnostics as well as in the understanding of structural changes leading to breast cancer.

It is still not certain whether the microcalcifications themselves induce or are related to the structural tissue changes that lead to the peak at $\mathrm{q}=1.725 \mathrm{~nm}^{-1}$, observed in malignant lesions. Whether the calcium deposits lead to tissue structural changes, or the tissue structural changes lead to calcium deposits, remains uncertain.

An important limitation of this study is the use of formalin for sample preparation, which can affect the collagen structure in the tissue and cause further degradation and dehydration, influencing SAXS structural analysis ${ }^{44}$.

As future work, we plan to measure fresh tissue samples, with and without microcalcifications, to remove the influence of formalin on collagen and combine all known 

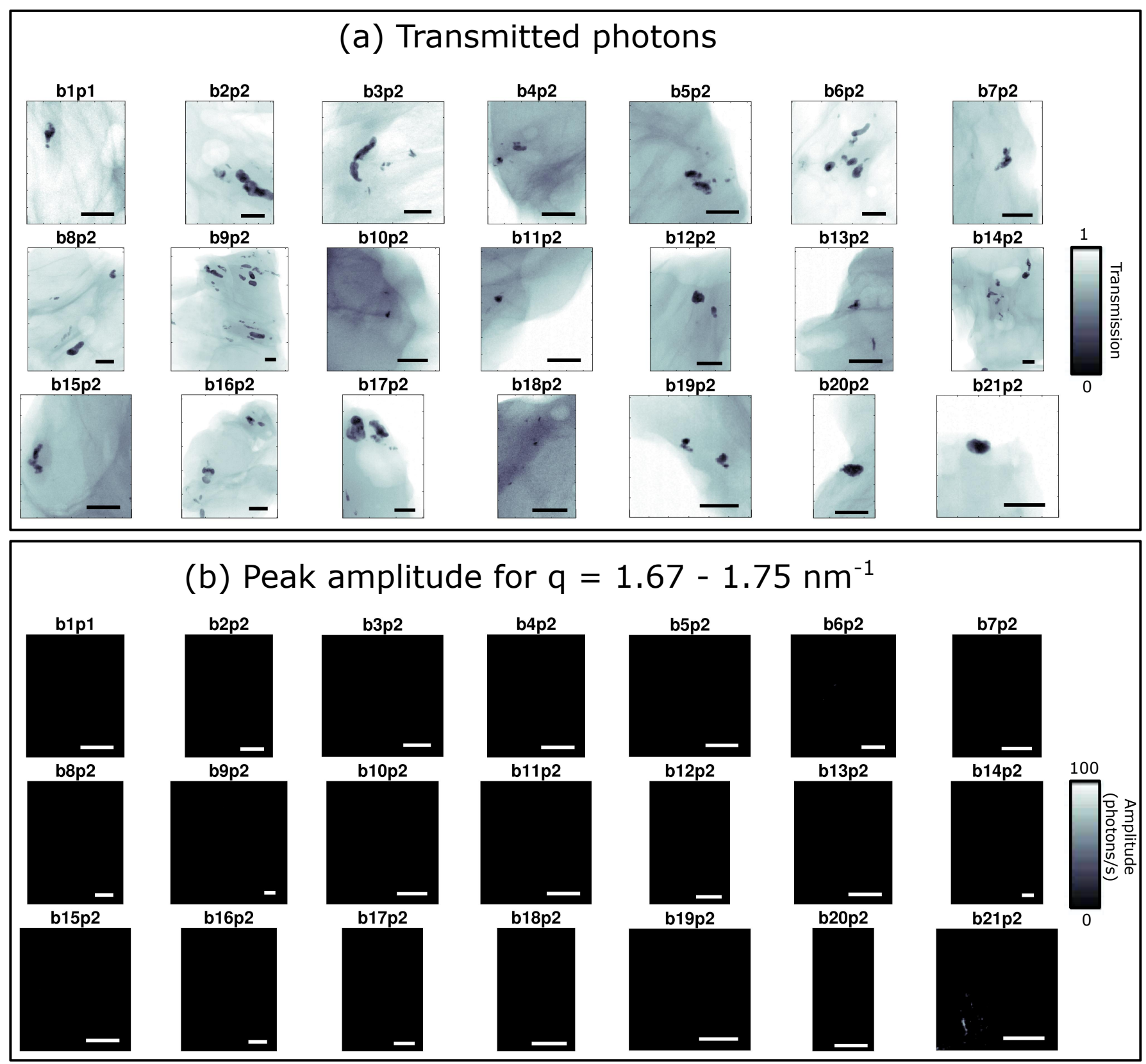

FIG. 2. Benign lesions measured by scanning SAXS: (a) Sample transmission maps. (b) Maps of the intensity of the peaks in the q-range $=1.67-1.75 \mathrm{~nm}^{-1}$. The scale bars corresponds to $1 \mathrm{~mm}$.

SAXS fingerprints, collagen, lipid, and the possible fi-407 brous tissue, for malignancy level assessment. Addition-408 ally, measurements of samples with type I microcalcifications, formed by calcium oxalate, in the q-range covered in this work, are essential to include the scattering ${ }_{409}$ of their crystalline configuration as an additional potential fingerprint for breast cancer classification. An interesting next step would be to measure the scattering ${ }^{410}$ signals of microcalcification-containing samples with $\mathrm{GI}^{41}$ which does not require a synchrotron and can be car- ${ }^{412}$ ried out with a regular X-ray tube, to test whether the $e^{413}$ information in the $q$ ranges accessible by this technique ${ }^{414}$ also allows a benign-malignant lesion discrimination and potentially a BI-RADS categorization ${ }^{4,5}$.

\section{ACKNOWLEDGMENT}

The authors acknowledge the European Research Council (ERC) (ERC-2012-StG 310005-PhaseX) and the Swiss National Foundation (SNF)-Sinergia CRSII2154472 MedXPhase and CRSII5-183568 for funding this work. They also thank Dr. Andreas Menzel from the the cSAXS beamline at the Swiss Light Source for his critical 

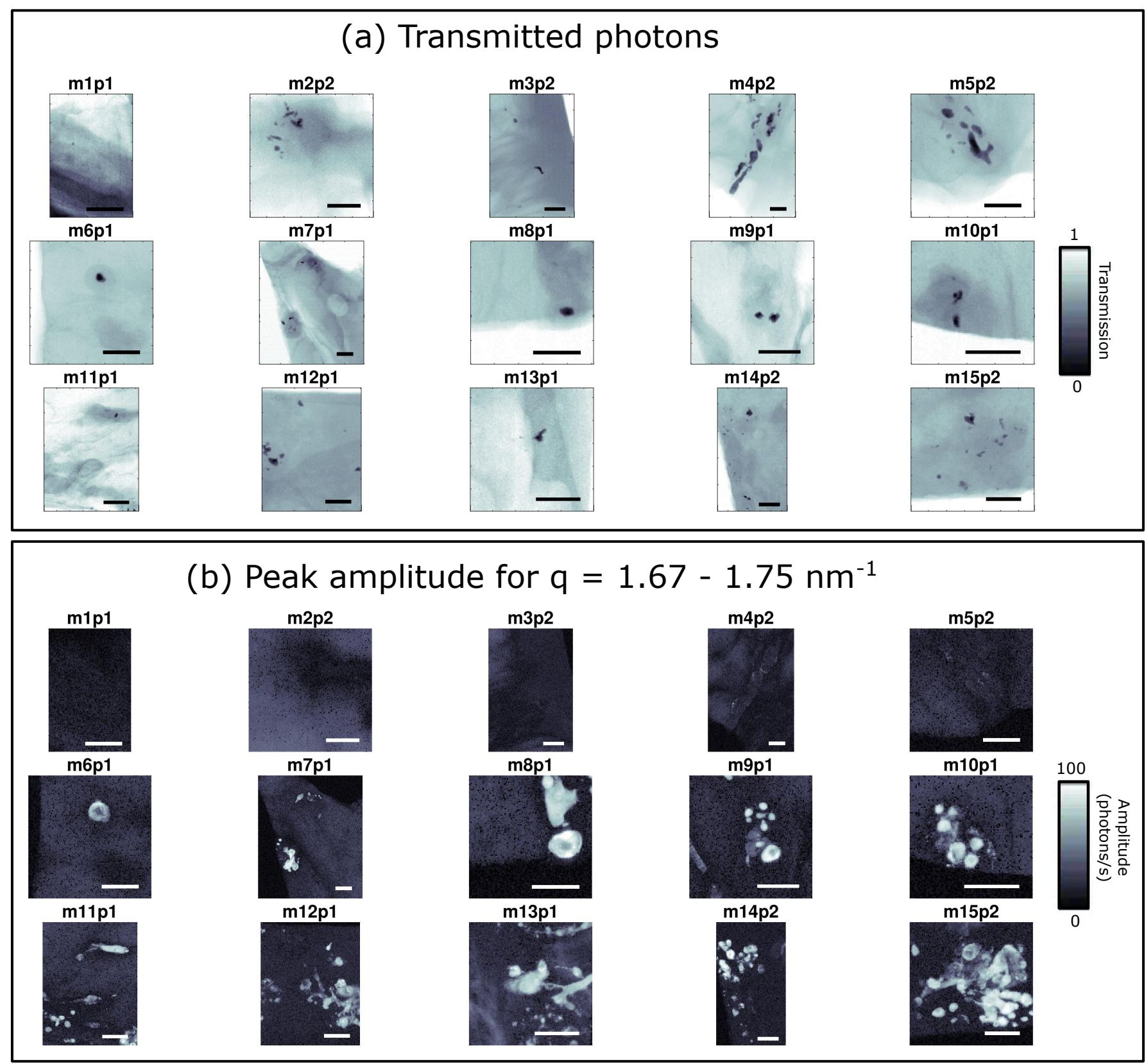

FIG. 3. Malignant lesions measured by scanning SAXS: (a) Maps of the relative transmitted photons. (b) Maps of the intensity of the peaks in the q-range $=1.67-1.75 \mathrm{~nm}^{-1}$. The scale bars corresponds to $1 \mathrm{~mm}$.

comments on the manuscript.

${ }^{1}$ Breast cancer statistics. Available from internet. ${ }^{431}$ http://www.wcrf.org/int/cancer-facts-figures/ 432 data-specific-cancers/breast-cancer-statistics (visited ${ }^{433}$ 07.08.2017)

${ }^{2}$ DOrsi CJ et al 2003 Breast Imaging Reporting and Data System ${ }^{435}$ (BI-RADS): ACR BI-RADSMammography 4th edn (American ${ }^{436}$ College of Radiology)

${ }^{3}$ Michel $\mathrm{T}$ et al 2013 On a dark-field signal generated by ${ }^{438}$ micrometer-sized calcifications in phase-contrast mammography ${ }^{439}$ Phys. Med. Biol., 58(8) 2713

${ }^{4}$ Scherer K et al 2015 Toward Clinically Compatible Phase- ${ }^{441}$ Contrast Mammography PLOS ONE 10(6) e0130776
${ }^{5}$ Wang Z, Hauser N, Singer G, Trippel M, Kubik-Huch R, Schneider C and Stampanoni M 2014 Non-invasive classification of microcalcifications with phase-contrast X-ray mammography Nat. Comm. 5 1-9

${ }^{6}$ Wang Z, Hauser N, Singer G, Trippel M, Kubik-Huch R, Schneider C and Stampanoni M 2016 Correspondence: Reply to Quantitative evaluation of X-ray dark-field images for microcalcification analysis in mammography Nat. Comm. 710868

${ }^{7}$ Glatter, Otto, and Otto Kratky, eds. Small angle X-ray scattering. Academic press, 1982

${ }^{8}$ Narayanan T 2009 High brilliance small-angle X-ray scattering applied to soft matter Current opinion in colloid and interface Science 14(6) 409-415 


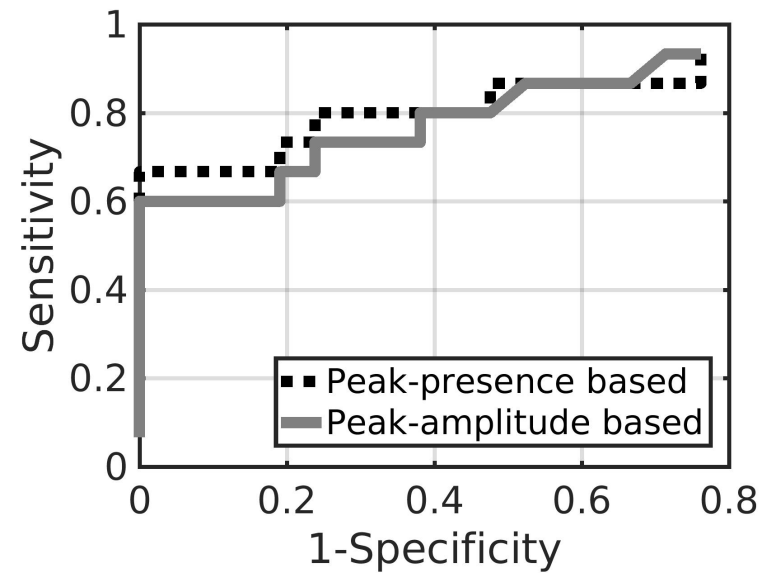

FIG. 4. Receiver operating characteristic (ROC) curves using 505 the presence of peaks (dotted line) or the amplitude of the ${ }^{506}$ peaks (solid line) as a criterion to classify benign and malig- ${ }^{507}$ nant breast tissue lesions.

${ }^{28}$ Castro CRF, Barroso RC and Lopes RT 2005 Scattering signa-

${ }^{29}$ Hajian-Tilaki K 2013 Receiver Operating Characteristic (ROC)

${ }^{9}$ Bunk O, Bech M, Jensen TH, Feidenhans R, Binderup T, Menzel ${ }_{513}^{512}$ A and Pfeiffer F 2009 Multimodal x-ray scatter imaging New J. ${ }_{514}^{513}$ Phys. 11(12) 123016

${ }^{10}$ Fernandez M et al 2005 Human breast cancer in vitro: matching ${ }_{516}$ histo-pathology with small-angle x-ray scattering and diffraction ${ }_{517}$ enhanced x-ray imaging Phys. Med. Biol. 50(13) 2991

${ }^{11}$ Fratzl P, Jakob HF, Rinnerthaler S, Roschger P and Klaushofer ${ }_{519}^{518}$ K 1997 Position-resolved small-angle X-ray scattering of complex ${ }_{520}$ biological materials J. Appl. Crystallogr. 30(5) 765-769

${ }^{12}$ Gourrier A, Wagermaier W, Burghammer M, Lammie D, Gupta ${ }_{522}^{521}$ HS, Fratzl P, Riekel C, Wess TJ and Paris O 2007 Scanning X-ray ${ }_{523}$ imaging with small-angle scattering contrast Applied Crystallog- ${ }_{524}$ raphy $\mathbf{4 0}$ (s1) s78-s 82

${ }^{13}$ Paris O, Loidl D, Peterlik H, Mueller M, Lichtenegger $\mathrm{H}$ and ${ }_{526}^{525}$ Fratzl P 2000 The internal structure of single carbon fibers deter- ${ }_{527}$ mined by simultaneous small-and wide-angle scattering $J . A p p l .{ }_{528}^{527}$ Crystallogr. 33(3) 695-699

${ }^{14}$ Rinnerthaler S, Roschger P, Jakob HF, Nader A, Klaushofer $\mathrm{K}_{530}^{529}$ and Fratzl P 1999 Scanning small angle X-ray scattering analysis ${ }_{531}$ of human bone sections Calcified tissue international 64(5) $422-{ }_{532}$ 429

${ }^{15} \mathrm{Siu} \mathrm{KKW}$ et al 2005 Identifying markers of pathology in $\mathrm{SAXS}_{534}^{533}$ data of malignant tissues of the brain Nuclear Instruments and $d_{535}$ Methods in Physics Research Section A: Accelerators, Spectrom- ${ }_{536}$ eters, Detectors and Associated Equipment 548(1) 140-146 ${ }_{536}^{536}$

${ }^{16}$ Giannini C et al 2014 Scanning SAXS-WAXS microscopy on ${ }_{538}^{537}$ osteoarthritis-affected bonean age-related study J. Appl. Crys ${ }^{538}$ tallogr. 47(1) 110-117

${ }^{17}$ Ryan EA and Farquharson MJ 2007 Breast tissue classification ${ }_{541}^{540}$ using $x$-ray scattering measurements and multivariate data anal- ${ }_{542}$ ysis Phys. Med. Biol. 52(22) 6679

${ }^{18}$ American College of Radiology (ACR). Breast imaging report- ${ }_{544}^{543}$ ing and data system (BI-RADS), breast imaging atlas. 4th ed., ${ }_{545}^{544}$ Reston, Va, Am College Radiology, 1259 (2003)

${ }^{19}$ Winchester DP, Jeske JM and Goldschmidt RA 2000 The diag- ${ }^{546}$ nosis and management of ductal carcinoma in situ of the breast $_{548}$ Am. Cancer J. Clin. 50(3) 184

${ }^{20}$ Schreer I and Luttges J 2001 Breast cancer: early detection Eur ${ }_{550}^{549}$ J. Radiol. 11 (Suppl 2) S307-S314

${ }^{21}$ Stephen A and Feig MD 2000 Ductal carcinoma in situ. Impli- ${ }_{552}$ cations for screening mammography Radiol. Clin. N. Am. 38(4) $)_{553}^{552}$ 653-668

${ }^{22}$ Henrich B, Bergamaschi A, Broennimann C, Dinapoli R, Eiken- ${ }_{555}{ }^{554}$ berry EF, Johnson I, Kobas M, Kraft P, Mozzanica A and $5375-5380$

${ }^{27}$ Sidhu S, Falzon G, Hart SA, Fox JG, Lewis RA and Siu KKW 2011 Classification of breast tissue using a laboratory system for small-angle x-ray scattering (SAXS) Phys. Med. Biol. 56(21) tures for some human tissues using synchrotron radiation $X$-Ray Spectrometry 34 477-480 Curve Analysis for Medical Diagnostic Test Evaluation Caspian Journal of Internal Medicine 4(2) 627-635

Schmitt B 2009 PILATUS: A single photon counting pixel detector for X-ray applications Nuclear Instruments and Methods tectors and Associated Equipment 607(1) 247-249

${ }^{3}$ CSH Protocols; 2008; doi:10.1101/pdb.prot4986

Frappart L et al 1984 Structure and composition of microcalcifimicroprobe analysis, and X-ray diffraction Hum. Pathol. 15(9) An overlooked form of microcalcification associated with benign breast disease Archives of pathology laboratory medicine 113(12) 1367-1369

Haka AS, Shafer-Peltier KE, Fitzmaurice M, Crowe J, Dasari RR and Feld MS 2002 Identifying microcalcifications in benign and malignant breast lesions by probing differences in their chemical Kiricuta IC, Simplaceanu V 1975 Tissue water content and nuclear magnetic resonance in normal and tumor tissues Cancer Res. 35 1164-1167

${ }^{31}$ Thakur SB, Bartella L, Ishill NM, Morris EA, Liberman L, Dershaw DD, Hricak H, Koutcher JA, Huang W 2006 Comparisons of water-to- fat ratios in malignant, benign breast lesions, and normal breast parenchyma: an in vivo proton MRS study Proceedings of the 14th Annual Meeting of ISMRM, Seattle

${ }^{32}$ Thakur SB, Bartella L, Ishill NM, Morris EA, Liberman L, Dershaw DD, Hricak H, Koutcher JA, Huang W 2006 Discrimination of choline- positive invasive breast carcinomas using water-to-fat ratio: a proton MRS study Proceedings of the 14 th Annual Meeting of ISMRM, Seattle

${ }^{33}$ Jagannathan NR, Singh M, Govindaraju V, Raghunathan P, Coshic O, Julka PK, Rath GK 1998 Volume localized in vivo proratio in patients receiving chemotherapy NMR Biomed. $11414-$ 422

${ }^{34}$ Sharma U, Kumar M, Sah RG and Jagannathan NR 2009 Study of normal breast tissue by in vivo volume localized proton MR spectroscopy: variation of water-fat ratio in relation to the heterogeneity of the breast and the menstrual cycle Magn. Reson. Imaging 27 785-791

${ }^{35}$ Pucci Minafra I, Luparello C, Sciarrino S, Tomasino RM and Minafra S 1985 Quantitative determination of collagen types present in the ductal infiltrating carcinoma of human mammary gland Cell Biology International Reports 9 291-296

${ }^{36}$ Pucci Minafra I, Minafra S, Tomasino RM, Sciarrino S and Tinrhous) carcinoma of the human breast. A possible role played by type I trimer collagen on the invasive growth Journal of Submicroscopic Cytology 18 795-805

${ }^{37}$ Rajkumar L, Balasubramanian K, Arunakaran J, Govindarajulu $\mathrm{P}$ and Srinivasan N 2005 Influence of estradiol on mammary tumour collagen solubility in DMBA-induced rat mammary tumours Cell Biology International 30 164-168

${ }^{38}$ Suhonen H, Fernndez M, Serimaa R and Suortti P 2005 Simulation of small-angle $\mathrm{x}$-ray scattering from collagen fibrils and comparison with experimental patterns Phys. Med. Biol. 50 54015416

${ }^{39}$ A.R. Round, "Ultra-structural analysis of breast tissue", Cranfield University, Cranfield, United Kingdom (2006). light microscopy, transmission and scanning electron microscopy, ton MR spectroscopy of breast carcinoma: variation of water-fat ervia R 1986 Collagen changes in the ductal infiltrating (scir- 
${ }^{40}$ Lewis RA, Rogers KD, Hall CJ, Towns-Andrews E, Slawson S,566 Evans A, Pinder SE, Ellis IO, Boggis CRM, Hufton AP and567 Dance DR 2000 Breast cancer diagnosis using scattered X-rays568 J. Synchrotron Radiat. 7 348-352

${ }^{41}$ Fernandez M, Keyrilainen J, Serimaa R, Torkkeli M, Karjalainen-570 Lindsberg ML, Tenhunen M, Thomlinson W, Urban V and571 Suortti P 2002 Small-angle x-ray scattering studies of human572 breast tissue samples Phys. Med. Biol. 47 577-592

${ }^{42}$ Fernandez M, Keyrilainen J, Karjalainen-Lindsberg ML, Leidenius M, Von Smitten K, Fiedler S and Suortti P 2004 Human breast tissue characterisation with small-angle X-ray scattering Spectroscopy 18 167-176

${ }^{43}$ Changizi V, Oghabian MA, Speller R, Sarkar S and Kheradmand AA 2005 Application of small angle x-ray scattering (SAXS) for differentiation between normal and cancerous breast tissue International Journal of Medical Sciences 2 118-121

${ }^{44}$ Sidhu S 2009 Small Angle X-ray Scattering as a Diagnostic Tool for Breast Cancer (Monash: Monash University) 